\title{
Présence d'anticorps antibovipestiques chez le dromadaire du Tchad
}

\author{
Y. MAURICE, A. PROVOST et C. BORREDON
}

\begin{abstract}
RÉSUMÉ
Les auteurs ont montré, en utilisant les réactions d'inhibition de l'hémagglutinine du virus de la rougeole et de séroneutralisation du virus bovipestique en culture de cellules, la présence d'anticorps contre le virus de la peste bovine dans les sérums de dromadaires du Tchad.
\end{abstract}

\section{I. - INTRODUCTION}

La question de la réceptivité de l'espèce cameline à la peste bovine est un sujet discuté et même controversé. Les opinions varient avec les observations cliniques des différents auteurs; il est intéressant de rappeler les constatations de ceux-ci avant d'exposer le résultat de nos recherches.

Dans son tralté sur les maladies du chameau, CURASSON (5) signale des observations, d'ailleurs non confirmées, selon lesquelles lors de la grande épizootie de 1892 qui frappa le cheptel bovin des confins sahariens les chameaux du Niger présentèrent une forme atténuée de peste bovine dans laquelle il n'y avait que fière, inappétence, larmoiement, jetage et une forme grave se rapprochant de la maladie bovine avec diarrhée, urine de couleur rouge et toux fréquente. TARTAKOWSKI en 1899 (20) démontre la sensibilité de ces animaux à la peste en inoculant 2 dromadaires et 4 chameaux du Turkestan : 2 présentèrent une réaction insignifiante ef 4 des symptômes de peste; un seul succombe. VEDERNIKOFF (22) et TSCHEGIS (21) observent des cas de peste chez le chameau de la région

* Communication présentée au $18^{\mathrm{e}}$ congrès mondial vélérinaire, Paris, 17-22 juillet 1967. de Bakou où sévit en 1898 une grosse épizootie. KOWALEVSKY (9) note également chez les chameaux des Kirghizes une maladie analogue à la peste bovine : les symptômes et les lésions observés par cet auteur sont rapportés en détail par CURASSON (5).

Par contre en 1897 HUTCHEON (8) fait remarquer que $\mathrm{KOCH}$ ne peut communiquer la peste bovine expérimentale au dromadaire ef en 1899 REFICK BEY (17) signale qu'au cours d'une épizootie de peste bovine en Turquie le chameau de la région s'est montré réfractaire à la maladie naturelle. En Inde en 1905, LINGARD (11) réalise diverses expériences faisanł conclure à la réceptivité du chameau : inoculant 5 animaux avec du sang virulent il constate, après une incubation de 5 à 7 jours, des symptômes divers qui suivent une poussée thermique initiale : vésicules et ulcères dans la bouche, diarrhée dans un seul cas, appétit conservé dans un seul cas. Chez certains l'urine est pigmentée et chez l'un des malades il y a des pustules cutanées. - Tous guérissent ef le sang de l'un d'eux, incculé à un autre bovin, provoque une peste bovine typique. Les abservations de CROSS en 1917 (4) vont dans le même sens : il remarque que de temps en temps des chameaux meurent de peste bovine dans les formations méharistes de l'Inde. Pour vérifier la susceptibilité des ani- 
maux, il inocule 3 chameaux âgés respectivement de 1 an, 6 ans et 12 ans, en même temps que 2 veaux témoins. Les 2 plus jeunes des chameaux présentent une peste typıque mals guérissent. Le $3^{\mathrm{e}}$ ne présente pas d'autres symptômes qu'un peu de diarrhée et une perte d'appétit puis se rétablıt lentement. CONTI (3) ef PRICOLO (15) en 1913, en Erythrée, constatent uré forme bénigne de peste chez le chameau et en 1932, en Inde, HAJl (7) signale des épizooties de peste bovine frappant les chameaux : les bovins conta. minateurs sont atteints d'une forme très pathogène et les symptômes chez les chameaux consistent en fièvre, abattement, diarrhée, ulcérations faisant suite d̀ une éruption vésiculaire sur les gencives, la langue, les lèvres. Par contre LEESE (10) dit avoir recherché vainement pendant 6 ans de prospection dans l'Inde la peste bovine chez le chameau. PECAUD (13) au Tchad, PIOT BEY (14) ef LITTLEWOOD (12) en Egypte, SAMARTSEU ef ARBUSOV (18) en Asie russe tiennent également l'espèce pour non réceptive. Plus récemment, DHILLON (6), en 1959, note de 1948 d' 195817 foyers de peste bovine chez le chameau dans le district de Hissar en Inde : 128 chameaux sont atteints dont 60 meurent. Au Kenya, en 1962, SCOTT et MAC DONALD (19) constatent que 30 chameaux du Nord Kenya qui avaient traversé la vague épizootique de 1962 n'ont pas conservé la trace d'anticorps sériques.

Devant tant de données contradictoires, il nous a paru intéressant de rechercher les anticorps antibovipestiques dans les sérums de dromadaires du Tchad, d'autant plus qu'aucune mention n'a été faite jusqu'ici dans ce pays sur d'éventuels cas de peste bovine chez le dromadaire alors que se manifestent tous les ans des foyers de cette maladie chez les autres ruminants.

\section{II. - TECHNIQUES}

L'enquête a été menée à l'aide de deux techniques :

10 Réaction d'inhibition de l'hémagglutinine

* Antigène Rougeole fourni par la maison Behringwerke AG, Marburg Lahn, Allemagne ; il s'agit d'une hémagglutinine commerciale préparée par action de l'éther éthylique et de tween 80. du virus de la rougeole* pour détecter la présence éventuelle d'anticorps inhibant l'hémagglutinine morbilleuse (en abréviation $=$ tesł l. $\mathrm{H}_{\text {. }}$ ).

La description de cette technique a fait l'objet d'une publication (1), aussi sera-t-il donné simplement un résumé de celle-ci. On met en contact dạns des tubes de Kahn $0,2 \mathrm{ml}$ de sérum aux dilutions $1 / 2,1 / 4,1 / 8 \ldots 1 / 1.024$ etc... avec 4 unités hémagglutinantes sous le volume de $0,2 \mathrm{ml}$ d'hémagglutinine morbilleuse non infectieuse. Les sérums ont été préalablement inactivés pendant une demi-heure à $56^{\circ} \mathrm{C}$ et adsorbés avec des globules rouges de singe Patas pour épuiser les hétéroagglutinines non spécifiques. Les dilutions de sérum et d'hémagglutinine sont faltes en tampon de COHEN. Après une heure de contact à $37{ }^{\circ} \mathrm{C}$, on ajoute au mélange $0,2 \mathrm{ml}$ d'une suspension au 1 : 250 d'hématies de singe (soit à 0,4 p. 100). La lecture de la réaction se fait après un séjour de 45 minutes à $37{ }^{\circ} \mathrm{C}$.

20 Réaction de séroneutralisation du virus bovipestique en cultures cellulaires, effectuée sur des sérums négatifs et sur des sérums positifs au test IH pour s'assurer de la présence effective d'anticorps spécifiques du virus bovipestique (en abréviation: test $S N$ ).

Les cultures de cellules sont réalisées à partir de reins d'embryons de veau. Les sérums sont dilués en progression de $1 / 2$ en 1/2 en tampon phosphate. La souche RPKO de Plowright et Ferris du virus pestique utilisée est à son $35^{\mathrm{e}}$ passage. Elle est diluée à partir d'une récolte de façon à contenir environ $10^{2} 2 \mathrm{D}$. C. $P_{50}$ par $\mathrm{ml}$. Des volumes égaux de virus et de sérums sont mélangés et laissés 1 heure à $37^{\circ} \mathrm{C}$ puis chacun d'eux est réparti dans les tubes de cultures de cellules (une partie de mélange pour 9 parties de milieu de culture). Les cultures de cellules sont examinées chaque jour pendant 12 jours.

Quelques remarques s'imposent à propos des techniques suivies ef de la valeur du test $\mathrm{IH}$. Celles-ci seront brièvement commentées, le détail de ces analyses devant faire l'objet d'une prochaine publication. La réaction d'inhibition de l'hémagglutınine du virus de la rougeole est rapide et facile à mettre en œuvre (1) (2) (16) ; c'est une méthode élégante de diagnostic de peste bovine qui a fait ses preuves à Farcha depuis deux ans. II ressort du travail effectué dans ce laboratoire sur la réaction d'inhibition de l'hémagglutination que son utilité est restreinte en 
grande partie à ce diagnostic. En effet il a été constaté avec des sérums de bovins qu'une dissociation s'installe dans le temps entre anticorps inhibant l'hémagglutination et anticorps neutralisant, les premiers pouvant disparaître alors que les seconds persistent. II s'ensuit que la réaction ne peut être employée pour effectuer des sondages d'immunité dans les troupeaux. En pays d'enzootie pestique, une réaction négative ne témoigne donc de la réceptivité au virus pestique qu'avec certaines restrictions. II est néanmoins possible d'utiliser le test $\mathrm{IH}$ pour déceler des anticorps antipestiques chez des animaux apparemment sains, étant bien entendu qu'il ne sera tenu compte que des résultats positifs, qui signeront la trace d'une infection récente due au virus pestique. Encore est-il qu'il sera difficilement possible de mesurer quantitativement l'état immunitaire de l'animal, et ceci d'autant plus que l'infection sera ancienne. C'est dans cette optique et avec ces restrictions qu'a été entreprise, en utilisant le test $\mathrm{IH}$, l'analyse de 482 sérums de dromadaires du Tchad tant pour détecter la présence d'anticorps antibovipestiques chez cet animal que pour s'assurer de la spécificité d'espèce de ce test, les sérums éventuellement positifs étant alors analysés par la méthode de séroneutralisation en culture de tissus.

\section{III. - RÉSULTATS ET DISCUSSION}

Contre toute attente une certaine proportion des sérums étudiés a présenté des anticorps inhibant l'hémagglutinine morbilleuse. Le détail des résultats est rapporté dans le tableau 1. Onze sérums sur 482 étudiés présentent des traces d'anticorps; 37 sur 482 soit 7,70 p. 100 sont nettement positifs : 22 au $1: 2,13$ au $1: 4$ et 2 au $1: 8$.

Ce résultat était à priori étonnant, car le dromadaire n'est pas, tout au moins en Afrique, considéré comme étant une espèce réceptive au virus bovipestique. II convenait donc de s'assurer de la spécificité des anticorps détectés. Ainsi 24 sérums positifs au test $\mathrm{IH}$ et 22 sérums négatifs à ce même test ont été analysés par séroneutralisation en cultures cellulaires. Les résultats obtenus sont rapportés dans le tableau II. On peut constater que :

10 Les 24 sérums positifs au test IH le sont également au test SN. Les dilutions ont été poussées pour 10 de ces sérums : les faux d'anticorps présents sont relativement faibles (1/64 au maximum). La spécificité de la réaction de séroneutralisation en matière de peste bovine permet d'af-

TABLEAU $\mathrm{N}^{\circ} \mathrm{I}$

Test d'inhibition de 1 'hëmagglutınine morbilleuse avec des sérums de dromadaires tchadiens

\begin{tabular}{|c|c|c|c|c|c|c|c|c|}
\hline \multirow[t]{2}{*}{$r$} & \multirow[b]{2}{*}{ Total } & \multicolumn{7}{|c|}{ Orígine géographique des sérums } \\
\hline & & Fort-Lamy & Massakory & Moussoro & Nokou & Z1gueï & Mao & Abëctié \\
\hline Nombre de sérums analysés & 482 & 72 & 1 & 129 & 23 & 34 & 130 & 93 \\
\hline $\begin{array}{l}\text { Nombre de sérums présentant } \\
\text { des traces d'anticorps à la } \\
\text { dilution } \frac{1}{2}\end{array}$ & 11 & 4 & 0 & 3 & 1 & 1 & 2 & 0 \\
\hline $\begin{array}{l}\text { Nombre de sérums positifs } \\
\text { au } \frac{1}{2}\end{array}$ & 22 & 5 & 0 & 11 & 2 & 2 & 2 & 0 \\
\hline $\begin{array}{l}\text { Nombre de sêrums positifs } \\
\text { au } \frac{1}{4}\end{array}$ & 13 & 6 & 0 & 4 & 0 & 0 & 2 & I \\
\hline $\begin{array}{l}\text { Nombre de sêrums positifs } \\
\text { au } \frac{1}{8}\end{array}$ & 2 & 2 & 0 & 0 & 0 & 0 & 0 & 0 \\
\hline $\begin{array}{l}\text { Nombre de sérums positifs } \\
\text { au } \frac{1}{16}\end{array}$ & 0 & 0 & 0 & 0 & 0 & 0 & 0 & 0 \\
\hline
\end{tabular}




\section{TABLEAU $\mathrm{N}^{\circ} \mathrm{II}$}

Corrélation entre le test d'Inhíbition de 1'hẻmagglutination et la séroneutralisation.

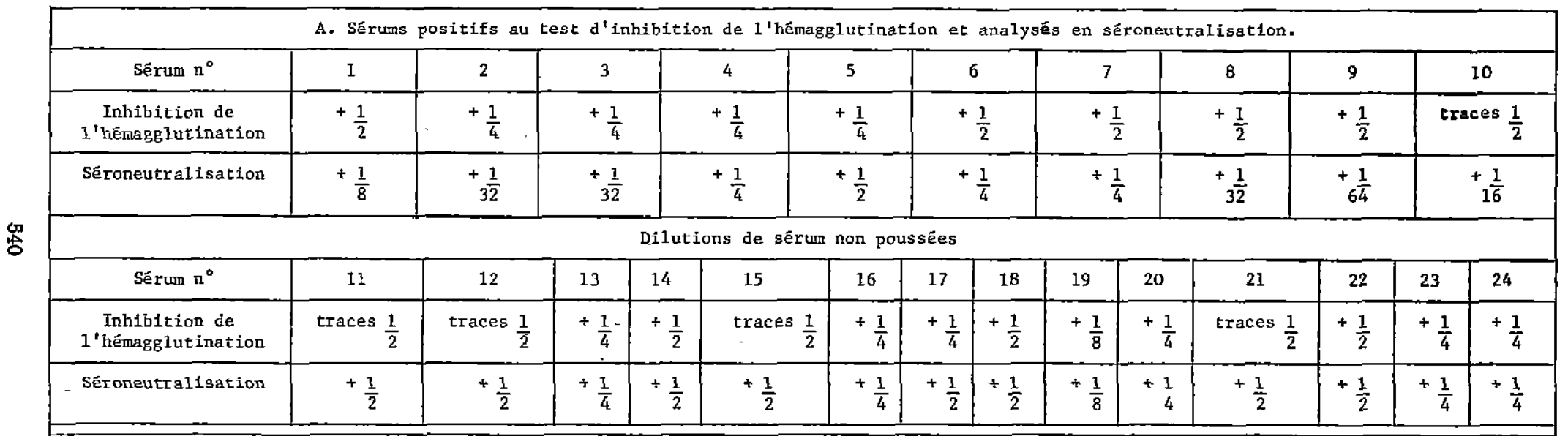

B. Sêrums négatifs au test d'inhibltion de 1 'hếmagglutination et analysés en sêtoneutralisation.

(18 sërưns nëgatifı au test IH ëgalement nêgatifs en séroneutralisation.)

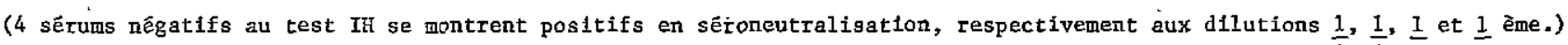


firmer qu'il s'agit d'authentiques anticorps antibovipestiques.

$2^{\circ}$ II n'existe pas de concordance quantitative entre anticorps inhibant l'hémagglutination et anticorps neutralisant. Ainsi les sérums 4 et 5 qui inhibent l'hémagglutination morbilleuse aux dilutions $1 / 4$ neutralisent le virus pestique dans les conditions de la réaction aux dilutions $1 / 4$ et 1/2. Par contre les sérums 8 et 9 qui inhibent l'hémagglutination au $1 / 2$ neutralısent respectivement le virus pestique aux dilutions $1 / 32$ et $1 / 64$.

3o If n'existe pas non plus de concordance qualitative entre anticorps inhibant l'hémagglutination et anticorps neutralisant. En effet, sur les 22 sérums négatifs au test $1 \mathrm{H}, 4$ présentent cependant des anticorps neutralisant. L'un de ces sérums neutralise même le virus à la dilution 1/64.

Ces résultats sont une illustration des restrictions apportées précédemment ò la valeur du test $\mathrm{IH}$, en particulier en cas de résultat négatif. Compte tenu de ce dernier résultat, il est donc vraisemblable que le pourcentage exact de sérum positif est bien supérieur à 7,70 p. 100 .

\section{IV. - CONCLUSIONS}

Etant donné l'extrême étendue du spectre de réceptivité à la peste bovine chez les ruminants, on devait se demander si le dromadaire d'Afrique était réceptif à cette virose. Les résultats obtenus ici sont intrinsèquement intéressants car ils démontrent d'une manière non équivoque qu'il en est bien ainsi sans que l'on puisse pour autant affirmer pour l'instant que la peste soit une composante pathologique de l'espèce cameline. La rareté des observations cliniques à ce sujet chez le dromadaire d'Afrique laisse plutôt à penser que la réceptivité du dromadaire à la peste bovine est faible. II est possible d'ailleurs qu'il puisse être touché sous une forme inapparente.

Il serait également intéressant de préciser le rôle de cette espèce dans l'écologie du virus : est-ce un relais ou un cul de sac ? Autant de questions à éclaircir dans les années à venir.

Instifut d'Elevage et de Médecine
vétérinaire des Pays tropicaux.
Laborotolre de Farcho. Fort Lamy.
Tchad.

Institut d'Elevage et de Médecine vétérinaire des Pays tropicoux.

Tchad.

\title{
SUMMARY
}

Presence of antibodies against Rinderpest virus in Dromedary in Chad

The presence of antibodies against Rinderpest virus has been evidenced in the sera of dromedary in Chad, by use of $\mathrm{H}$. I. Test with measles virus and seroneutralisation of Rinderpest virus in cell cultures.

\section{RESUMEN}

Presencia de anticuerpos ántibovipésticos en el dromedario del Chad

\begin{abstract}
Utilizando las reacciones de inhibición de la hemaglutinina del virus del sarampión y de seroneutralización del virus bovipestico en cultivo de células, los autores mostraron la presencia de anticuerpos contra el virus de la peste bovina en los sueros de los dromedarios del Chad.
\end{abstract}

\section{BIBLIOGRAPHIE}

1. BÖGEL (K.), ENDERS-RUCKLE (G.), PROVOST (A.). - Une réaction sérologique rapide de mesure des anticorps anfibovipestiques. C.R. Acod. Sci., Paris, 1964, 259 : 482.

2. BÖGEL (K.), PROVOST (A.), ENDERSRUCKLE (G.). - Hemagglutinations Hemmungsreaktion mit Masernantigen bei
Rinderpest. I. Anwendung in der Diagnostic. II. Antikórperproduktion nach Inokulation verschiedener Lebendimpfstoffe beim Rind. Zentbl. Bokt., 1966, $199: 1-19$ et 201 : 137-152.

3. CONTI (G.). - Moderno Zooiatro, Torino, $1913,24: 215$.

4. CROSS. - 1917. Cité par Curasson (5). 
5. CURASSON (G.). - Le chameau et ses maladies, Paris, Vigot Frères, 1947, p. 57.

6. DHILLON (S.S.). - Incidence of Rinderpest in Hissar district. Ind. vet. J., 1959, 36: 603.

7. HAJI (C. S. G.), - Indian vet. J., 1932, $9: 13$.

8. HUTCHEON. - 1897. Cité par Curasson (5).

9. KOWALEVSKY. - Cité par Curasson (5).

10. LEESE (A. S.). - A treatise on the OneHumped Camel in Health and in Disease. Stamford: Haynes, 1927, p. 272.

11. LINGARD (A.), - Report on the preparation of Rinderpest serum, Calcutta, 1905. Résumé ds Bull. Inst. Past., 4 : 235.

12. LITTLEWOOD (W.). - - J. Comp. Path., 1905, $18,312$.

13. PECAUD (G.). - Contribution à l'étude de la pathologie vétérinaire de la colonie du Tchad. Bull. Soc, Path. Exot., 1924, 17 : 196-207.
14. PIOT BEY, - 1920. Cité par Curasson (5).

15. PRICOLO. - 1913. Cité par Curasson (5).

16. PROVOST (A.), BOGEL (K.), BORREDON (C.). - Une nouvelle méthode sérologique rapide d'identification du virus bovipestique. C. R. Acad. Scl. Paris, 1964, $259: 684$.

17. REFICK BEY, - 1899. Cité par Curasson (5).

18. SAMARTSEU (A. A.) et ARBUZOV (P. N.). - Réceptivité du chameau à la morve, à la peste bovine et à la péripneumonie. Veterinariyo, Moscou. Analyse ds Vet. Bull., $1945,15: 396$.

19. SCOTT (G. R.), MAC DONALD (J.).Kenya Camels and Rinderpest. Bull. Epiz. Dis. Afric., 1962, $10: 495-497$.

20. TARTAKOWSKY (M. M.). - Arch. Sci. biol. St Petersburg, 1899, $8: 11$.

21. TSCHEGIS. - 1902. Cité par Curasson (5).

22. VEDERNIKOFF, -1902 . Cité par Curasson (5). 